\title{
Avaliação do fluxo de referência para um centro de especialidades odontológicas implantado em cidade de médio porte na região Sudeste
}

\author{
Evaluation of the referece flow for a center for dental specialties \\ deployed in a midsize city in Brazilian southeast
}

\author{
Luciana Afonso Rodrigues ${ }^{1}$ Jane Darley Martins Vieira², Isabel Cristina Gonçalves Leite ${ }^{3}$
}

\begin{abstract}
Resumo
A organização, pelos gestores da saúde, de um adequado sistema de referência à atenção especializada ainda representa um dos mais importantes desafios a serem enfrentados e evidencia, no cotidiano dos serviços, a busca por uma assistência integral aos cidadãos. Foi avaliado o fluxo de referência dos usuários que acessam os serviços prestados pelo Centro de Especialidades Odontológicas (CEO) implantado em cidade de médio porte da Região Sudeste brasileira. Foi feita análise quantitativa pautada na qualidade dos registros encontrados em prontuários e formulários de referência utilizados na unidade, perfazendo um total de 409 prontuários referentes aos meses de setembro e outubro de 2011. Os resultados encontrados foram: prontuários completos: 71,4\% para endodontia, 62,9\% para cirurgia e diagnóstico de câncer bucal e 74\% para periodontia. Prontuários legíveis: 74,3\% para endodontia, 72\% para cirurgia e diagnóstico de câncer bucal e 84,3\% para Periodontia. Prontuários com indicação de tratamento especializado em conformidade com os critérios ministeriais preconizados: $62,1 \%$ para endodontia, 59,4\% para cirurgia e diagnóstico de câncer bucal e 77,2\% para periodontia. A organização do sistema de referência e contrarreferência representa um dos eixos básicos para uma assistência integral à saúde.
\end{abstract}

Palavras-chave: Política Nacional de Saúde; saúde bucal; Sistema Único de Saúde; assistência integral à saúde.

\begin{abstract}
Organization, for health managers, of an adequate referral system to specialized care still represents one of the most important challenges faced and evidences, in everyday services, the search for a full assistance to citizens. The flow of users who access the specialized care provided by Centers for Dental Specialties (CEO) deployed a midsize city in southeast Brazilian was evaluated.. The study is the type of documents and descriptive. Quantitative analysis was made based on the quality of records found in medical records and forms used in the reference unit, a total of 409 records for the months of September and October 2011. The results were: to complete medical records: $71,4 \%$ for endodontics, $62,9 \%$ for surgery and diagnosis of oral cancer and $74 \%$ to periodontics. Legible records: $74,3 \%$ for endodontics, $72 \%$ for surgery and diagnosis of oral cancer and $84,3 \%$ to periodontics. Records indicating specialized treatment in accordance with the criteria established by the MS: $62,1 \%$ for endodontics, $59,4 \%$ for surgery and diagnosis of oral cancer and $77,2 \%$ to periodontics. Organizing the system of reference and counter reference is one of the basic pillar for a comprehensive health care.
\end{abstract}

Keywords: National Health Policy; oral health; Unified Health System; comprehensive health care.

Trabalho realizado no Centro de Especialidades Odontológicas Norte - Juiz de Fora (MG), Brasil.

'Especialista em Saúde Coletiva; Aluna de MBA de Gestão em Saude, Acreditação e Auditoria, Universidade Federal de Juiz de Fora (UFJF) - Juiz de Fora (MG), Brasil. 2Especialista em Vigilância Sanitária de Serviços de Saúde (2004) e Direito Sanitário (2006) pela Escola Nacional de Saúde Pública (FIOCRUZ) - Rio de Janeiro (RJ), Brasil ${ }^{3}$ Doutora em Saúde Publica pela FIOCRUZ - Rio de Janeiro (RJ); Docente da Faculdade de Medicina da Universidade Federal de Juiz de Fora (UFJF) - Juiz de Fora (MG), Brasil.

Endereço para correspondência: Luciana Afonso Rodrigues - Rua Pedro Scapin, 270/405 - São Mateus - CEP: 36025-120 - Juiz de Fora (MG), Brasil E-mail: luafonso@oi.com.br

Fonte de financiamento: nenhuma.

Conflito de interesse: nada a declarar. 


\section{INTRODUÇÃO}

Diversos fatores influenciaram o aumento da demanda por serviços de saúde nos últimos anos, no Brasil. Destacam-se o aumento da população nas grandes cidades e o próprio aumento da oferta desses serviços. Como consequência desses fatores, o setor saúde vive um momento de elevação de gastos, sobretudo no setor público; levando a questão do planejamento de ações ao centro das discussões entre gestores das três esferas de governo, com o objetivo de melhorar a qualidade desses serviços ${ }^{1}$.

No campo da saúde bucal, o panorama das desigualdades se apresenta de forma contundente, no que se refere ao acesso da população menos favorecida economicamente aos serviços públicos, além da pouca oferta de atenção especializada. Paralelamente, as iniciativas de avaliação dos serviços públicos de saúde bucal ainda se mostram insuficientes, em relação à qualidade, forma de acesso e referenciamento dos usuários ${ }^{2}$.

Vários estudos ${ }^{3-5}$ apontam os seguintes aspectos da qualidade desejáveis nos serviços de saúde em geral: efetividade, eficácia, eficiência, equidade, qualidade científico-técnica, acessibilidade, adequação e aceitação dos usuários. $\mathrm{Na}$ Odontologia, a busca por uma prestação de serviços públicos que atendam ao critério de continuidade, pautada no princípio da integralidade, ancora-se no entendimento e aplicação dos conceitos de referência e contrarreferência. A qualidade dos registros utilizados neste processo é fundamental para se avaliar a qualidade desses serviços ${ }^{3}$.

A referência é representada pelo fluxo, por meio de encaminhamento do paciente com necessidades de saúde complexas para centros de atenção especializada. O fluxo contrário, ou seja, o retorno do paciente ao nível da atenção primária próxima de sua residência é denominado contrarreferência. O estabelecimento de métodos para facilitar esse fluxo é primordial para que se faça um atendimento integral; porém, em todas as áreas da saúde, os métodos utilizados permanecem insuficientes, sobretudo no nível municipal ${ }^{6}$.

Diante desses desafios, o Ministério da Saúde (MS) implantou, a partir do ano de 2004, a Política Nacional de Saúde Bucal. São bases dessa política a ampliação da oferta de serviços especializados em saúde bucal com organização da atenção primária no nível local e o estabelecimento de diretrizes para o sistema de referência e contrarreferência ${ }^{7}$.

O objetivo primordial desta pesquisa foi a descrição de dados sobre a referência dos usuários que acessam os serviços prestados por Centro de Especialidades Odontológicas (CEO) localizado em município polo, que presta atendimento aos cidadãos de sua microrregião, e está situado na Região Sudeste brasileira. A avaliação foi direcionada à qualidade do preenchimento de prontuários e formulários de referência utilizados na unidade avaliada. Para que o processo de referência e contrarreferência de um determinado serviço ou rede de atenção à saúde seja eficaz, são necessários: a correta organização, a real utilização e entendimento desses instrumentos pelos trabalhadores do sistema e o adequado preenchimento dos prontuários e formulários existentes na organização ${ }^{8}$.

\section{METODOLOGIA}

O presente estudo atendeu aos requisitos da Declaração de Helsinque e às recomendações da Resolução no 196, de 16 de outubro de 1996, do Conselho Nacional de Saúde ${ }^{9}$, tendo sido aprovado pelo Comitê de Ética em Pesquisas em Seres Humanos da Universidade Federal de Juiz de Fora - MG, sob parecer $n^{\circ}$ 214/2011. Tratou-se de estudo documental e descritivo, no qual foi avaliado o fluxo e a organização da referência de usuários para o CEO Norte em Juiz de Fora, por meio da análise dos registros encontrados em prontuários e formulários de referência utilizados na unidade, nos meses de setembro e outubro de 2011.

O CEO Norte está credenciado pelo MS como CEO tipo II e funciona no mesmo espaço físico da Unidade Odontológica Regional Norte (UOR Norte), que presta atendimento em atenção primária aos usuários residentes na Região Sanitária 8 do município de Juiz de Fora. A UOR Norte recebe, em média, 180 pacientes/mês para tratamento odontológico em atenção primária. Desse total de pacientes, $30 \%$ são direcionados à atenção especializada. O CEO Norte foi implantado do ano de 2006, junto à UOR Norte, e tem, sob sua responsabilidade sanitária, a população residente na área urbana das Regiões Sanitárias 7 e 8 da cidade de Juiz de Fora (103.054 habitantes), área rural adjacente (2.600 habitantes), além da população residente em 11 municípios da microrregião (65.000 habitantes). As especialidades oferecidas no CEO Norte são: periodontia, endodontia, cirurgia bucomaxilofacial, diagnóstico de câncer bucal e odontopediatria.

As questões investigadas foram: o adequado preenchimento dos documentos utilizados para a referência de pacientes ao tratamento especializado prestado pelo CEO Norte e encaminhamento dos usuários com base nas recomendações previstas pelo $\mathrm{MS}^{10}$.

Foi realizada análise quantitativa dos dados coletados e as pesquisadoras realizaram a coleta de registros em conjunto. Inicialmente, foi realizada uma coleta experimental para concordância entre as pesquisadoras sobre a classificação dos documentos, de acordo com os critérios de avaliação.

Foram selecionados para o estudo os prontuários e formulários de referência pela atenção primária da UOR Norte, pela atenção primária prestada em Unidades de Atenção Primária 
à Saúde (UAPS) da região de abrangência do CEO Norte e pela atenção primária prestada pelos municípios pactuados da microrregião, sob responsabilidade sanitária da unidade avaliada. Foram listados todos os prontuários contidos nas agendas das especialidades de periodontia, endodontia e cirurgia bucomaxilofacial/diagnóstico de câncer bucal nos meses de setembro e outubro do ano de 2011, totalizando 409 prontuários avaliados. $\mathrm{O}$ critério adotado para a seleção dos meses foi a inexistência de datas atípicas para o acesso de usuários à unidade avaliada, como verificado nos meses de férias escolares, férias dos profissionais ou recessos, fato que não ocorreu nos meses de setembro e outubro do referido ano.

Os prontuários estavam arquivados em ordem numérica. Alguns prontuários constavam na agenda de uma ou mais especialidades. Cada prontuário selecionado teve seus dados descritos para todas as especialidades indicadas e ali relacionadas.

Foram excluídos todos os prontuários e formulários não referenciados à atenção especializada e os de referência à especialidade de Odontopediatria, uma vez que os pacientes são atendidos integralmente nas dependências da UOR/CEO NORTE, ou seja, passam também pela atenção primária dentro da unidade. Os prontuários e formulários referenciados para a especialidade de diagnóstico de câncer bucal estão inseridos na especialidade de cirurgia bucomaxilofacial, visto que o atendimento é prestado pelo mesmo profissional para as duas especialidades.

$\mathrm{O}$ atendimento a pacientes com necessidades especiais, no município de Juiz de Fora e na microrregião é prestado pelo Centro de Atendimento a Pacientes com Necessidades
Especiais (CEO COAPE), credenciado como CEO tipo II pelo MS. Essa unidade não foi objeto do presente estudo.

A Figura 1 representa o fluxograma de desenho da pesquisa. Como variáveis avaliadas foram considerados:

- Preenchimento adequado dos documentos citados: foram considerados adequados os documentos que atenderam a dois quesitos principais: legibilidade e completude. Para avaliar a legibilidade, foi utilizada escala baseada em classificações encontradas na literatura médica ${ }^{11,12}$. Dessa forma, os prontuários ou formulários poderiam ser classificados como:

- Legível: documento lido normalmente, que deixa claro todas as palavras, números, símbolos e abreviaturas;

- Pouco legível: documento que gerou algum tipo de dúvida pelo leitor quanto ao entendimento ou legibilidade de palavras, números, símbolos e abreviaturas, podendo ser deduzido ou interpretado mais de $50 \%$ de seu conteúdo (dados pessoais do paciente: nome completo, data de nascimento; número de cada elemento dentário ou sextante a ser tratado pelo especialista; motivo do encaminhamento ao CEO e assinatura do cirurgião-dentista que referenciou o paciente);

- Ilegível: documento que apresentou impossibilidade de entendimento do seu conteúdo, com, pelo menos, $50 \%$ de conteúdo indecifrável.

Quanto à completude, foram considerados:

- Completo: documento que apresentou todos os itens imprescindíveis para que o especialista pudesse prestar o

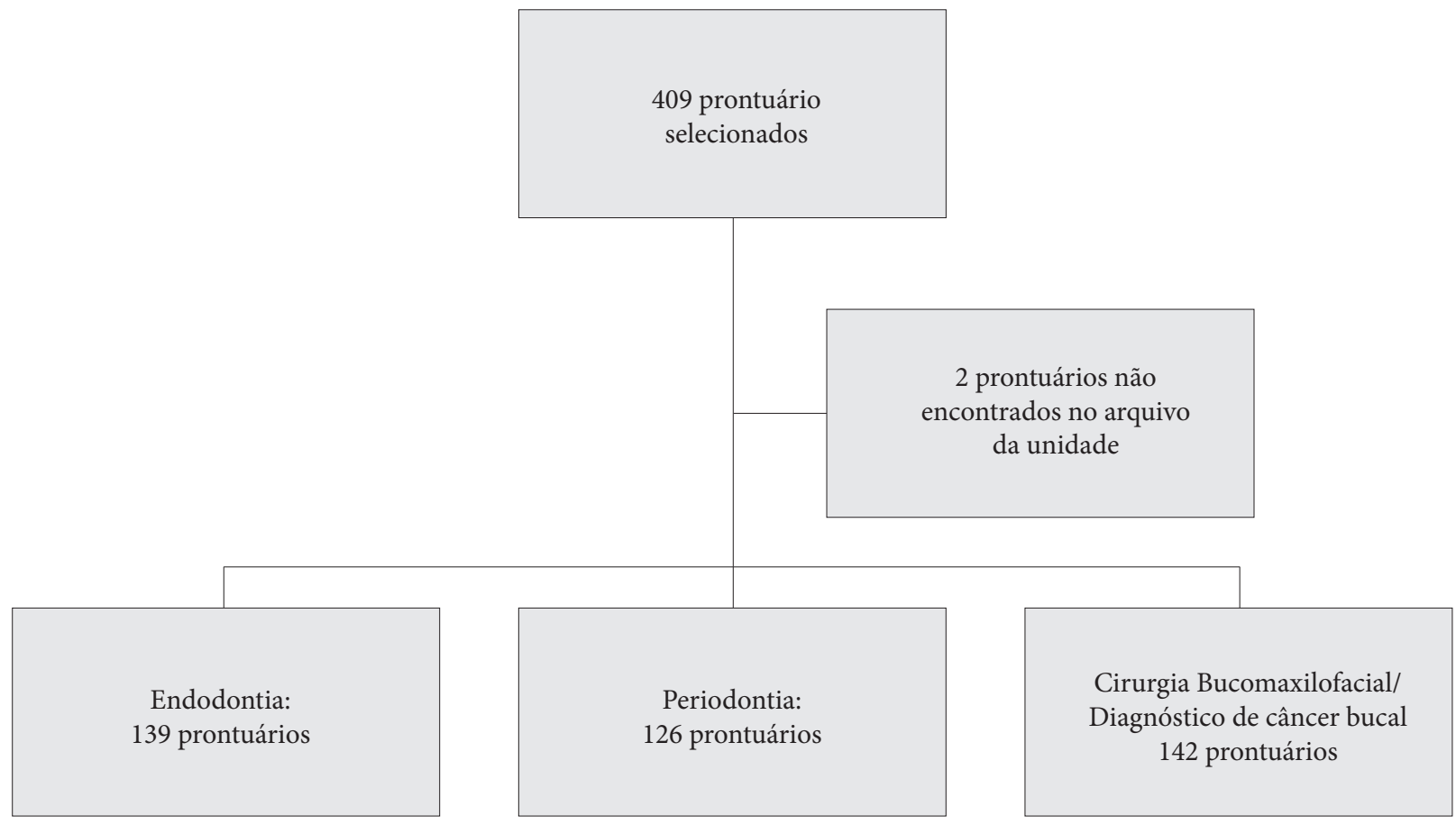

Figura 2. Fluxograma de desenho do estudo 
atendimento (dados pessoais do paciente: nome completo; data de nascimento; número de cada elemento dentário ou sextante a ser tratado pelo especialista; motivo do encaminhamento ao $\mathrm{CEO}$ e assinatura do cirurgião-dentista que referenciou o paciente);

- Incompleto: documento que não apresentou todos os itens relacionados.

Aplicação das Recomendações para referência aos Centros de Especialidades Odontológicas (BRASIL, 2004) ${ }^{13}$. Os prontuários ou formulários de referência foram considerados:

- Com indicação correta: documento cujo motivo do encaminhamento consta no protocolo preconizado pelo MS;

- Com indicação incorreta: documento que apresentou quaisquer outros motivos de encaminhamento não descritos no protocolo preconizado pelo MS.

O Programa SPSS, versão 15.0 (SPSS INCORPORATION, 2006), foi utilizado para entrada e análise descritiva dos dados (medidas de frequência e dispersão). A comparação de proporções entre os grupos foi feita pelo teste do $\chi^{2}$.

\section{RESULTADOS}

As Tabelas 1, 2 e 3 apresentam os resultados encontrados no estudo.

$\mathrm{Na}$ associação entre as variáveis utilizadas e origem dos pacientes, houve diferença significativa para as variáveis completude e legibilidade $(\mathrm{p}<0,01)$. Em relação aos critérios de referenciamento do MS não houve diferença significativa entre os grupos. A Tabela 4 descreve a completude, legibilidade e indicação para especialidades conforme recomendações do MS segundo procedência do prontuário.

\section{DISCUSSÃO}

A avaliação pode ser conceituada como a análise de um determinado processo produtivo ou programa, a partir de seus objetivos, com a finalidade de orientar ou redirecionar para a conquista de benefícios ${ }^{14}$.
Para Akermann e Nadanovsky ${ }^{4}$, é preciso estabelecer critérios de avaliação da qualidade dos serviços de saúde no sentido de ajuizar determinados resultados atingidos. A avaliação, contudo, não seria considerada um fim em si mesma, mas um processo em que um julgamento explícito é elaborado, e a partir daí desencadear-se-ia um movimento de transformação na direção da qualidade previamente desejada.

Existe uma tendência crescente na direção de estudos relacionados à avaliação das práticas de saúde. Os serviços prestados pelos Centros de Especialidades Odontológicas foram objeto de alguns estudos, ainda em pequeno número, mas que apontam na direção da instituição de protocolos de referência e contrarreferência, avaliação para qualidade da atenção e grau de satisfação de usuários ${ }^{7,15}$.

Em Juiz de Fora, atualmente, estão em funcionamento quatro CEOs. A proposta de política de saúde bucal implantada no município a partir do ano de 2000 prevê o fortalecimento e a reorganização da atenção primária além do incremento da atenção especializada, com a implantação das UORs ${ }^{16}$. Essas unidades abrigam, no mesmo espaço físico, assistência odontológica nos dois níveis, com o CEO funcionando junto à atenção primária, trazendo uma nova possibilidade de oferta de serviços de saúde bucal ancorada no princípio da integralidade. O trabalho multiprofissional e os ganhos em escala são exemplos dos benefícios

Tabela 1. Características dos prontuários segundo variável Completude. Juiz de Fora, MG, 2011

\begin{tabular}{|c|c|c|c|}
\hline $\begin{array}{l}\text { Variável / } \\
\text { Especialidade }\end{array}$ & $\begin{array}{c}\text { Prontuários } \\
\text { (n) }\end{array}$ & $\begin{array}{c}\text { Completos } \\
(\%)\end{array}$ & $\begin{array}{c}\text { Incompletos } \\
(\%)\end{array}$ \\
\hline Endodontia & 140 & 71,4 & 28,6 \\
\hline Cirurgia & 143 & 62,9 & 37,1 \\
\hline Periodontia & 127 & 74,0 & 26,0 \\
\hline
\end{tabular}

Tabela 2. Características dos prontuários segundo variável Legibilidade. Juiz de Fora, MG, 2011

\begin{tabular}{lcccc} 
Variável / & $\begin{array}{c}\text { Prontuários } \\
\text { Especialidade }\end{array}$ & $\begin{array}{c}\text { Legíveis } \\
\%\end{array}$ & $\begin{array}{c}\text { Pouco legíveis } \\
\%\end{array}$ & $\begin{array}{c}\text { Ilegíveis } \\
\%\end{array}$ \\
Endodontia & 140 & 74,3 & 25,7 & 0,0 \\
Cirurgia & 143 & 72,0 & 27,3 & 0,7 \\
Periodontia & 127 & 84,3 & 15,7 & 0,0 \\
\hline
\end{tabular}

Tabela 3. Características dos prontuários segundo variável Indicação para especialidades conforme recomendações do Ministério da Saúde. Juiz de Fora, MG, 2011

\begin{tabular}{lccc} 
Variável / Especialidade & $\begin{array}{c}\text { Prontuários } \\
\text { (n) }\end{array}$ & $\begin{array}{c}\text { Seguem recom. } \\
(\%)\end{array}$ & $\begin{array}{c}\text { Não seguem recom. } \\
(\%)\end{array}$ \\
Endodontia & 140 & 62,1 & 33,6 \\
Cirurgia & 143 & 4,3 & 27,3 \\
Periodontia & 127 & 59,4 & 13,3 \\
\hline
\end{tabular}

recom.: recomendação 
Tabela 4. Prontuários em conformidade com critérios avaliados conforme origem da referência. Juiz de Fora, MG, 2011

\begin{tabular}{lcccccc} 
Variável / Especialidade & $\begin{array}{c}\text { Prontuários } \\
(\mathbf{n})\end{array}$ & $\begin{array}{c}\text { UOR Norte } \\
(\%)\end{array}$ & $\begin{array}{c}\text { Prontuários (n) } \\
\text { UAPS }\end{array}$ & $\begin{array}{c}\text { UAPS } \\
(\%)\end{array}$ & $\begin{array}{c}\text { Prontuários (n) } \\
\text { Microrregião }\end{array}$ & $\begin{array}{c}\text { Microrregião } \\
\text { (\%) }\end{array}$ \\
Completude & 163 & 65,5 & 51 & 76,1 & 42 & 76,4 \\
Legibilidade & 180 & 72,3 & 55 & 82,1 & 49 & 89,1 \\
Indicação conforme MS & 162 & 65,1 & 42 & 62,7 & 44 & 80,0 \\
\hline
\end{tabular}

UOR: Unidade Odontológica Regional; UAPS: Unidade de Atenção primária à Saúde

alcançados. O município possui seus próprios prontuários e formulários de encaminhamento, assim como os municípios da microrregião.

Cabe ressaltar aspectos importantes referentes à regionalização e descentralização no campo da assistência à saúde. De acordo com a avaliação de Pestana e Mendes sobre o Pacto de Gestão ${ }^{17}$, o percurso proposto pelas Normas Operacionais Básicas para implementação de uma rede de serviços regionalizada e hierarquizada sofreu modificações ao longo do tempo de existência do Sistema Único de Saúde (SUS). A construção do modelo baseado na oferta de uma extensa gama de serviços municipais não alcançou êxito. Tornou-se necessária uma readaptação deste contexto, caminhando da municipalização autárquica em direção à regionalização cooperativa, com autossuficiência dos municípios polo das microrregiões no âmbito da atenção secundária.

O conceito de Sistema de Saúde advém de várias partes funcionando de forma independente, para o bem do todo, sendo substituído pela ideia de Redes de Atenção à Saúde (RAS). Organizar os serviços de saúde em redes de atenção significa proporcionar assistência integral centrada nos interesses dos usuários e proporcionar acesso e de forma desigual, na medida das necessidades dos que dele necessitam. Assim, as RAS só poderiam funcionar de forma efetiva, trazendo a ideia de "transversalidade" por todo o sistema de saúde $^{18}$. Para Mendes ${ }^{19}$, a integração vertical, nas RAS, se faz por meio de uma gestão única, baseada na comunicação fluida entre as diferentes unidades produtivas dessa rede; comandando, agregando todos os pontos de atenção, elaborando e operacionalizando um sistema logístico moderno e eficaz.

Nesse sentido, seria importante reorganizar a rede de serviços, dentro de cada microrregião, repensando-se a utilidade de prontuários diferenciados para cada município.

Neste estudo, verificou-se que, nos municípios pactuados da microrregião e nas UAPS da área de abrangência do CEO Norte, o preenchimento adequado dos registros foi superior ao da UOR Norte, adjacente ao CEO avaliado. Nos documentos avaliados, constavam de uma até três especialidades indicadas por paciente referenciado. $\mathrm{O}$ seu correto preenchimento e a adequada indicação às especialidades poderiam representar diminuição de gastos e de retornos desnecessários dos usuários à unidade de referência.

A completude e legibilidade dos prontuários (aqui considerados como conjunto de documentos indispensáveis ao atendimento odontológico e não apenas como ficha clínica), além do seu correto tempo de armazenamento e padronização das informações nele contidas, garantem ao paciente melhor qualidade nos serviços a ele prestados e representam proteção legal para o cirurgião-dentista ${ }^{20}$. É de grande importância o entendimento dos profissionais de saúde sobre os aspectos citados e seu constante treinamento para o preenchimento adequado da documentação que constitui o prontuário.

O prontuário eletrônico já é realidade em serviços hospitalares ${ }^{21}$ e se impõe como necessidade, viabilizando rapidez nas informações, apesar da obrigação legal de utilização do prontuário em papel. O desenvolvimento de prontuários padronizados baseados em sistemas digitais, a possibilidade de manter registros que acompanham a história clínica do paciente e a criação de bases de dados com amplitude de informações trazem um grande impacto na melhoria da eficácia, eficiência, segurança, e qualidade da prática de saúde ${ }^{22}$. A possibilidade de gerenciar um sistema digitalizado de informações, com gestão regional compartilhada pelos municípios envolvidos, poderia representar um grande diferencial na organização da rede de atenção à saúde nas cidades polo.

A resolutividade da assistência prestada no nível das UAPS poderá reduzir a demanda por consultas especializadas e exames, especialmente os de maior complexidade, reservando os recursos públicos para garantir os procedimentos realmente necessários. Parte dos encaminhamentos feitos por profissionais da rede básica a especialistas não esgotam todos os recursos assistenciais disponíveis na atenção primária ${ }^{23}$.

Desse modo, pode-se conceituar integralidade como modo de organizar o processo de trabalho em saúde, de forma a otimizar o seu impacto epidemiológico, articulando atenção à demanda espontânea com a oferta programada de atenção à saúde, ou; como busca contínua de ampliar a satisfação das necessidades de um grupo populacional com ampliação da eficiência ${ }^{13}$. 


\section{CONSIDERAÇÕES FINAIS}

Este estudo pretendeu avaliar aspectos do cotidiano dos serviços prestados pelos Centros de Especialidades Odontológicas. A implantação dos CEOs propiciou a concretização da assistência especializada em saúde bucal no SUS, preenchendo uma lacuna de décadas.

O caminho a se percorrer, em direção a uma prática pautada na integralidade, passa pela educação continuada dos profissionais envolvidos na prestação dos serviços e implantação de protocolos clínicos adequados à realidade dos usuários. A adoção de prontuários padronizados, e elaborados de forma a simplificar o preenchimento, é de grande importância para a melhoria da comunicação entre os diversos níveis de atenção.

São necessários outros estudos na área de gestão em saúde bucal coletiva para auxiliar na melhoria da qualidade dos serviços prestados à população. Espera-se que os resultados obtidos contribuam com a assistência prestada pelos Centros de Especialidades Odontológicas já implantados.

\section{REFERÊNCIAS}

1. Zucchi P, Del Nero C, Malik AM. Gastos em saúde: os fatores que agem na demanda e na oferta dos serviços de saúde. Saúde Soc. 2000;9(1/2):127-50.

2. Barros AJD, Bertoldi AD. Desigualdades na utilização e no acesso a serviços odontológicos: uma avaliação em nível nacional. Ciênc saúde coletiva. 2002;7(4):709-17.

3. Acurcio FA, Cherchiglia ML, Santos MA. Avaliação de qualidade de serviços de saúde. Saúde Debate. 1991;33:50-3.

4. Akerman M, Nadanovsky P. Avaliação dos serviços de saúde: avaliar o quê? Cad saúde pública. 1992;8(4):361-5.

5. Vuori, H. A qualidade da saúde. Divulg saúde debate. 1991;(3):17-24.

6. Fratini JRG, Saupe R, Massaroli A. Referência e contra-referência: contribuição para a integralidade em saúde. Cienc Cuid Saúde. 2008;7(1):65-72.

7. Chaves SCL, Barros SG, Cruz DN, Figueiredo ACL, Moura BLA, Cangussu MCT. Política Nacional de Saúde Bucal: fatores associados à integralidade do cuidado. Rev saúde pública. 2010;44(6):1005-13.

8. Silva AM, Vargas AMD, Ferreira EF, Abreu MHNG. A integralidade da atenção em diabéticos com doença periodontal. Ciênc saúde coletiva. 2010;15(4):2197-206.

9. Conselho Nacional de Saúde (Brasil). Resolução n. 196, de 10 de outubro de 1996. Aprova as diretrizes e normas regulamentadoras de pesquisas envolvendo seres humanos. Diário Oficial da União: 1996; 16 out.

10. Brasil. Ministério da Saúde. Recomendações para referência e contrareferência aos Centros de Especialidades Odontológicas. In: Cadernos de Atenção Básica, n. 17. Brasília: Ministério da Saúde; 2006. p. 70-92.

11. Aguiar G, Silva Jr LA, Ferreira MAM. Ilegibilidade e ausência de informação nas prescrições médicas: fatores de risco relacionados a erros de medicação. RBPS. 2006;19(2):84-91.

12. Rosa MB, Perini E. Erros de medicação: quem foi? Rev Assoc Med Bras. 2003;49(3):335-41.

13. Brasil. Ministério da Saúde. Integralidade da Atenção à Saúde. Brasília: Ministério da Saúde; 2004.
14. Donabedian A. Enfoques básicos para la evaluación. In: Donabedian A. La calidad de la atencion medica: definicion y metodos de evaluacion. Cidade do México: La prensa Médica Mexicana; 1984. p. 97-157.

15. Figueiredo N, Góes PSA. Construção da atenção secundária em saúde bucal: um estudo sobre os Centros de Especialidades Odontológicas em Pernambuco, Brasil. Cad saúde pública. 2009;25(2):259-67.

16. Prefeitura de Juiz de Fora. Plano Municipal de Saúde 2010-2013 [cited 2011 April 21]. Available from: http.www.pjf.mg.gov.br

17. Minas Gerais. Pacto de Gestão: da municipalização autárquica à regionalização cooperativa. Belo Horizonte: Secretaria de Estado da Saúde; 2004.

18. Cecílio LCO. As necessidades de saúde como conceito estruturante na luta pela integralidade e eqüidade na atenção à saúde. In: Pinheiro R, Mattos RA. Os sentidos da integralidade na atenção e no cuidado à saúde. Rio de Janeiro: IMSUERJ-ABRASCO; 2001. p. 113-26.

19. Mendes EV. As redes de atenção à saúde: Revisão bibliográfica, fundamentos, conceito e elementos constitutivos. In: Mendes EV. As redes de atenção à saúde. Brasília: Organização Pan-Americana da Saúde; 2011. p. 61-208.

20. Ramos DIA. Prontuário Odontológico: Aspectos Éticos e Legais. [dissertação]. Piracicaba, SP: Faculdade de Odontologia de Piracicaba, Universidade Estadual de Campinas; 2005.

21. Pires FA, Furuie SS, Gutierrez MA, Tachinardi U. Prontuário eletrônico: aspectos legais e situação atual [cited 2011 Jul 14]. São Paulo: Instituto do Coração (InCor)/HC-FMUSP; 2003. Available from: http://telemedicina. unifesp.br/pub/SBIS/CBIS2004/trabalhos/arquivos/476.pdf

22. Massad E, MarinHF, Azevedo Neto RS. O prontuário eletrônico do paciente na assistência, informação e conhecimento médico. São Paulo: H. de F. Marin; 2003.

23. Franco BT, Magalhães Jr MH. Integralidade na assistência à saúde: a organização das linhas do cuidado. In: Merhy EE. Magalhães Jr HM, Rimoli J, Franco TB, Bueno WS. O Trabalho em saúde: olhando experienciando o SUS no cotidiano. São Paulo: Hucitec; 2006. p.125-34.

Recebido em: 19/03/2012 Aprovado em: 21/01/2013 